\title{
Aphakic macular oedema: a two-year follow-up study
}

\author{
R. A. HITCHINGS \\ From the Department of Clinical Ophthalmology, Institute of Ophthalmology, Moorfields Eye Hospital, London
}

SUMMARY A 2-year follow-up study was carried out on patients known to have developed aphakic macular oedema 6 weeks postoperatively, and the results were compared with those in a control group who had not developed oedema. Four eyes $(12 \%)$ still had macular oedema, the visual acuities ranging between $6 / 5$ and 6/9. There was no significant difference in visual acuities at 2 years between the 2 groups of patients. Visual acuity had significantly improved between 6 weeks and 2 years in the eyes that had macular oedema at 6 weeks after extraction. Factors causing persistence of aphakic macular oedema are discussed.

Macular degeneration following cataract extraction was described by Irvine (1953). Gass and Norton (1966) described perifoveal capillary leakage of fluorescein and aphakic macular oedema in patients referred because of a fall in visual acuity noted 4 to 12 weeks after cataract extraction. Prospective studies indicated that aphakic macular oedema occurred in $40 \%$ (Irvine et al., 1971), 50\% (Hitchings et al., 1975), and 60\% (Yoshioka and Kawashima, 1971) of patients, many of whom maintained good vision.

Gass and Norton (1969) showed that in patients referred with visual loss from this condition the oedema settled within 6 months in $50 \%$ and persisted for 3 years in $8 \%$. In a prospective study Hitchings et al. (1975) showed that at 6 months resolution had occurred in $57 \%$ and decreased fluorescein leakage in $28 \%$, while $15 \%$ where unchanged or worse.

This paper reports the results of a 2-year follow-up study on patients who participated in a prospective study of the incidence of aphakic macular oedema (Hitchings et al., 1975) and assesses the effects of macular oedema on visual acuity and macular appearance.

\section{Materials and methods}

All patients who participated in the original study were asked to return for a full ophthalmic examination. They had undergone cataract extraction 2 to $2 \frac{1}{2}$ years before. Examination included refraction, slit-lamp biomicroscopy of the anterior and posterior

Address for reprints: R. A. Hitchings, FRCS, Department of Clinical Ophthalmology, Moorfields Eye Hospital, City Road, London ECIV 2PD segment, and colour and fluorescein photography. The angiographic technique employed was the standard technique used at Moorfields Eye Hospital (Teeters and Bird, 1973).

\section{Results}

Seventy-one patients were asked to return. Seven patients had died, 2 were too ill to attend, and 1 had developed a rhegmatogenous retinal detachment in the follow-up period. Six patients could not be traced. Fifty-five patients, 57 eyes (of which 33 eyes had shown aphakic macular oedema at 6 weeks postoperatively) attended for the follow-up study.

Aphakic macular oedema was observed in 4 eyes, the visual acuities being $6 / 5,6 / 6$, and 2 eyes with 69. A comparison of visual acuities 6 weeks and $2+$ years for each of these eyes is seen in Table 1. It will be seen that acuity had improved in each eye.

A comparison of visual acuities at 6 weeks and 2 to $2 \frac{1}{2}$ years after lens extraction of patients who did and did not show fluorescein leakage at 6 weeks is seen in Figs. 1 and 2. It will be seen that, while at 6 weeks the mean visual acuity is $6 / 7 \cdot 5$ and $6 / 6$ respectively for the eyes that did and did not show fluorescein leakage, at $2+$ years the mean visual acuity is virtually the same, at $6 / 6$ for the 2 groups. Chi-square analysis demonstrated a significant difference in visual acuity at 6 weeks and $2+$ years in those patients' eyes that developed aphakic macular oedema $(P<0.05)$ but no significant difference over the same period for the eyes that did not. Nor was there any significant difference between the 2 groups at 2 weeks or $2+$ years. It will be noted that 4 of the eyes had visual acuities of less than $6 / 18$ because of 1 eye each with amblyopia, marked postoperative astigmatic error, retinoschisis 
Table 1 Visual acuities and complicating features in eyes with aphakic macular oedema 2 to $2 \frac{1}{2}$ years after operation

\begin{tabular}{llll}
\hline Eye No. & VA at 6 weeks & VA at 2 to $2 \frac{1}{2}$ years & Complication \\
\hline 1 & $6 / 6$ & $6 / 5$ & Heterochromic cyclitis \\
2 & $6 / 12$ & $6 / 6$ & - \\
3 & $6 / 24$ & $6 / 9$ & Persistent flat anterior chamber \\
4 & $6 / 12$ & $6 / 9$ & Vitreous loss \\
\hline
\end{tabular}

affecting the macula, and congenital cataract. These patients have not been included in the calculations of mean visual acuity.

The anterior hyaloid face had ruptured in 29 eyes, of which 15 had shown aphakic macular oedema 6 weeks postoperatively. No patient had inflammatory cells in the vitreous.

Fine granular pigmentation in the macular region was seen in 10 eyes, of which 9 had shown leakage of fluorescein. One eye known to have developed aphakic macular oedema now had disciform degeneration affecting the macular region.
Multiple drusen at the posterior pole were noted in 2 eyes not known to have developed aphakic macular oedema. The appearances of the macular region at 2 to $2 \frac{1}{2}$ years after extraction is summarised in Table 2.

\section{Discussion}

On the basis of the patients described aphakic macular oedema is a common sequela of uncomplicated cataract extraction (Hitchings et al., 1975). Characteristic features are a fall in visual
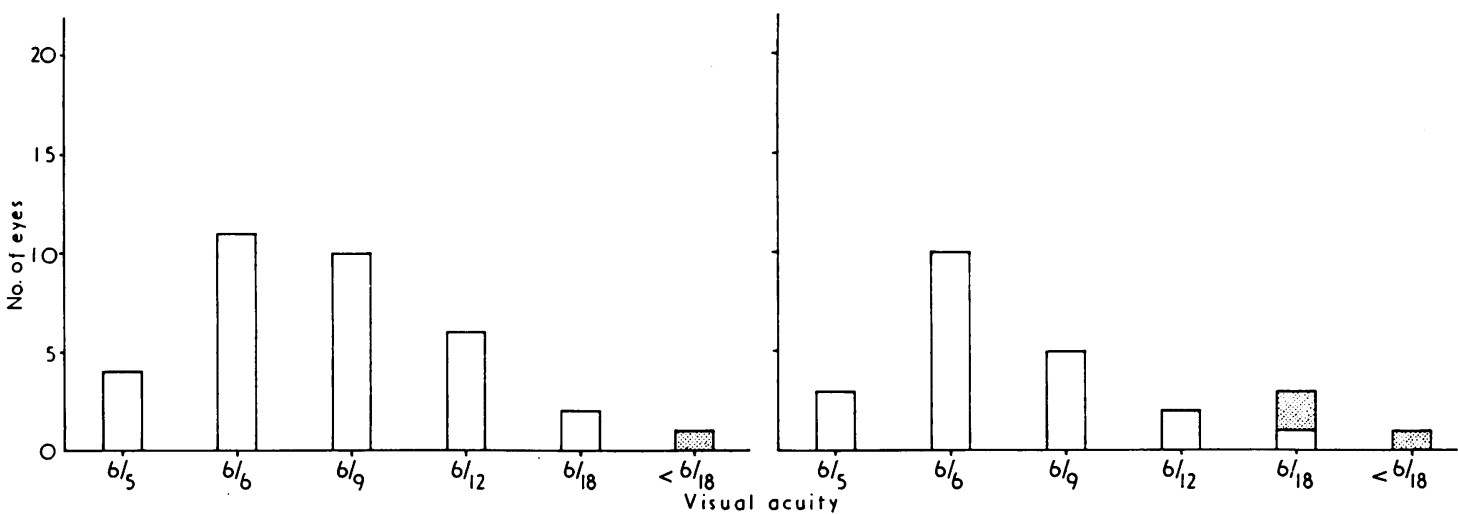

Fig. 1 Comparison of visual acuities at 6 weeks in eyes with and without cystoid macular oedema at 6 weeks after cataract extraction. NB: Stippling denotes eyes not included in calculation of mean visual acuity-see text

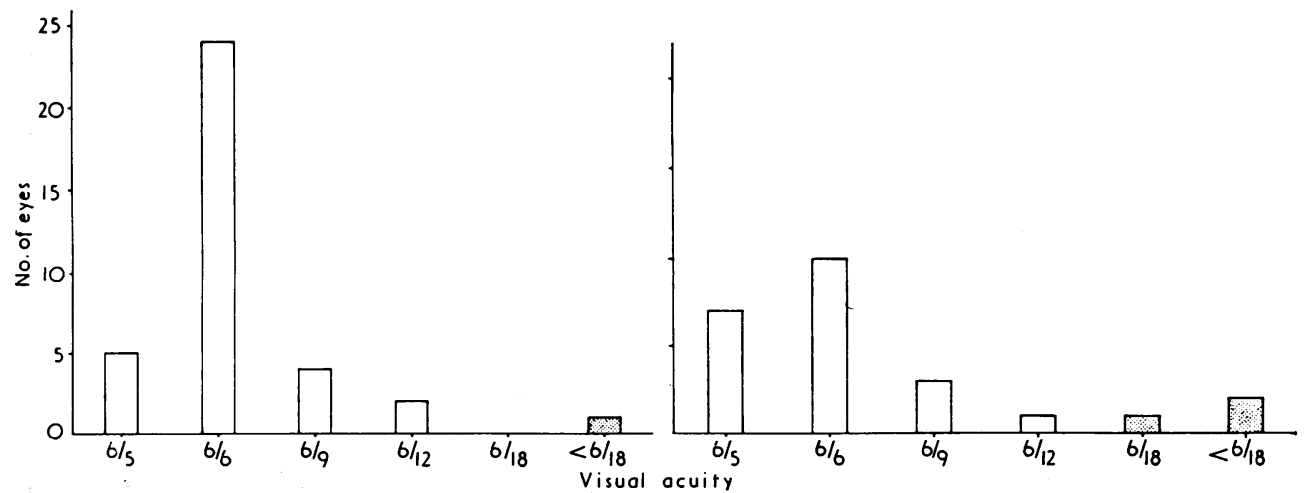

Fig. 2 Comparison of visual acuities at 2 to $2 \frac{1}{2}$ years in eyes with and without cystoid macular oedema at 6 weeks after cataract extraction. NB: Stippling denotes eyes not included in calculation of mean visual acuity-see text 
Table 2 Appearance of the macular region 2 to $2 \frac{1}{2}$ years after extraction

\begin{tabular}{llll}
\hline A. Eyes never shown to develop oedema & & B. Eyes shown to have oedema at 6 weeks after operation \\
& No. of eyes & No. of eyes \\
\hline Pigmentary stippling & 1 & Pigmentary stippling & 9 \\
Retinoschisis affecting posterior pole & 1 & Disciform degeneration & 1 \\
Macular drusen & 2 & Macular oedema & 4 \\
\hline
\end{tabular}

acuity or a failure to achieve expected levels of visual acuity in the immediate postoperative period. Biomicroscopy reveals cells in the posterior vitreous and a loss of foveal reflex; cystoid intraretinal spaces may be seen in the macular region. Fluorescein angiography reveals intraretinal accumulation of fluorescein in a 'petalloid' pattern.

The aphakic macular oedema seen in the patients reported here appeared to undergo spontaneous resolution. Fluorescein leakage completely ceased by 6 months in 16 of the 29 eyes studied (Hitchings et al., 1975). Four eyes $(12 \%)$ of those showing macular oedema at 6 weeks after extraction still had macular oedema at 2 to $2 \frac{1}{2}$ years. In 3 of these eyes fluorescein angiography at 6 months showed macular oedema. No other factor considered likely to be responsible for the macular oedema was identified. Specifically, no patient had been receiving topical adrenaline therapy (Michels and Maumanee, 1975), and no eyes had evidence of a retinal vein occlusion, diabetic retinopathy, or posterior segment inflammation. Complications identified for the eyes with persistent aphakic macular oedema are shown in Table 1. Similar complications, however, occurred in patients in whom oedema was never detected.

Perifoveal pigmentary stippling was the only visible change found preferentially in eyes known to have aphakic macular oedema. Gass and Norton (1969) noted 'pigment mottling' in the macular region after resolution of aphakic macular oedema. In our patients this morphological change did not appear to affect the visual acuity.

The mean visual acuity for the eyes known to have developed aphakic macular oedema improved, so that by 2 to $2 \frac{1}{2}$ years there was no significant difference between eyes known to have and not to have developed macular oedema. Visual acuities ranging from $6 / 5$ to $6 / 9$ were obtained in the 4 eyes with persistent macular oedema. In these eyes prolonged oedema was compatible with good acuity, perhaps because none had developed irreversible structural changes, noted by Gass and Norton (1969). The good acuity seen with persistent macular oedema is in marked contrast to the poor acuity seen in eyes with macular oedema associated with a central or branch vein occlusion or diabetic retinopathy.
These contrasting visual acuities may reflect a difference in composition of the oedema fluid and/or the adequacy of retinal nutrition.

In most instances aphakic macular oedema is transient, producing little if any long-term effect on vision. The condition is important when the oedema is prolonged; intraretinal structural changes with decreased acuity may eventually occur (Gass and Norton, 1969). Factors causing development and then persistence of aphakic macular oedema still remain obscure. While aphakic macular oedema is a common sequel to uncomplicated cataract extraction, its persistence may reflect a non-specific retinal response to a variety of stimuli. It must be assumed that an unidentified factor or combination of factors caused aphakic macular oedema to persist over a 2-year period in these eyes.

I thank consultants at Moorfields Eye Hospital for allowing me to study patients under their care. I thank Mr A. C. Bird for helpful discussion, $\mathrm{Mr} \mathrm{H}$. Donovan for statistical analysis, and Miss E. Nightingale, who typed the manuscript.

\section{References}

Gass, J. D. M., and Norton, E. W. D. (1966). Cystoid macular edema and papilledema following cataract extraction. Archives of Ophthalmology, 76, 646-661.

Gass, J. D. M., and Norton, E. W. D. (1969). Follow-up study of cystoid macular edema following cataract extraction. Transactions of the American Academy of Ophthalmology and Otolaryngology, 73, 665-682.

Hitchings, R. A., Chisholm, I. H., and Bird, A. C. (1975). Aphakic macular edema: Incidence and pathogenesis. Investigative Ophthalmology, 14, 68-71.

Irvine, S. R. (1953). A newly defined vitreous syndrome following cataract surgery. American Journal of Ophthalmology, 36, 599-619.

Irvine, A. R., Bresky, R., Crowder, B. M., Forster, R. K., Hunter, D. M., and Kulvin, S. M. (1971). Macular edema after cataract extraction. Annals of Ophthalmology, 3, 1234-1240.

Michels, R. G., and Maumanee, A. E. (1975). Cystoid macular edema associated with topically applied epinephrine in aphakic eyes. American Journal of Ophthalmo$\log$, 80, 379-388.

Teeters, V. W., and Bird, A. C. (1973). The development of neovascularisation of senile macular degeneration. American Journal of Ophthalmology, 76, 1-18.

Yoshioka and Kawashima (1971). Macular edema following cataract extraction. Transactions Japanese Society of Ophthalmology, 75, 2269-2273. 